\title{
Study of CVD diamond layers with amorphous carbon admixture by Raman scattering spectroscopy
}

\author{
Anna Dychalska ${ }^{1}$, Piotr Popielarski ${ }^{2}$, Wojciech Franków ${ }^{2}$, KAZIMIERz Fabisiak ${ }^{2,3}$, \\ KAZIMIERZ PAPROCKI ${ }^{2}$, MirosŁaW SZYBOWICZ ${ }^{1 *}$ \\ ${ }^{1}$ Faculty of Technical Physics, Poznan University of Technology, Piotrowo 3, 60-965 Poznań, Poland \\ ${ }^{2}$ Institute of Physics, Kazimierz Wielki University, Powstańców Wielkopolskich 2, 85-090 Bydgoszcz, Poland \\ ${ }^{3}$ Medical Physics Department, Oncology Center, I. Romanowskiej 2, 85-796 Bydgoszcz, Poland
}

\begin{abstract}
Raman spectroscopy is a most often used standard technique for characterization of different carbon materials. In this work we present the Raman spectra of polycrystalline diamond layers of different quality, synthesized by Hot Filament Chemical Vapor Deposition method (HF CVD). We show how to use Raman spectroscopy for the analysis of the Raman bands to determine the structure of diamond films as well as the structure of amorphous carbon admixture. Raman spectroscopy has become an important technique for the analysis of CVD diamond films. The first-order diamond Raman peak at ca. $1332 \mathrm{~cm}^{-1}$ is an unambiguous evidence for the presence of diamond phase in the deposited layer. However, the existence of non-diamond carbon components in a CVD diamond layer produces several overlapping peaks in the same wavenumber region as the first order diamond peak. The intensities, wavenumber, full width at half maximum (FWHM) of these bands are dependent on quality of diamond layer which is dependent on the deposition conditions. The aim of the present work is to relate the features of diamond Raman spectra to the features of Raman spectra of non-diamond phase admixture and occurrence of other carbon structures in the obtained diamond thin films.
\end{abstract}

Keywords: Raman spectroscopy; carbon materials; diamond structure; amorphous carbon; CVD diamond films

(C) Wroclaw University of Technology.

\section{Introduction}

Diamond films can be grown using a variety of chemical vapor deposition (CVD) techniques. The most popular methods are: microwave plasma CVD (MP CVD), hot filament CVD (HF CVD), radio frequency CVD (RF CVD), laser induced plasma (LIP) and flame methods [1].

Diamond is valued not only as a gem, but also technologically as an extraordinary material with unique physical, chemical, and optical properties. Due to its well-known high hardness, singular strength, high thermal conductivity, low coefficient of thermal expansion, chemical inertness, excellent optical transparency, and semiconductor properties, it has attracted intensive scientific studies and technological interest worldwide [2,3].

\footnotetext{
*E-mail: miroslaw.szybowicz@ put.poznan.pl
}

Diamond is chemically inert and does not react with common acids even at elevated temperatures. A large number of industrial applications take advantage of diamond's excellent properties coupled with extreme strength and chemical inertness $[4,5]$ over other materials for harsh conditions [6].

The optimization of control parameters of CVD growth process is a very important task to get diamond layer having properties suitable for specific application. In the HF CVD method we can distinguish 18 parameters, which control the growth process, among the others: the process pressure, temperature of filament, temperature of substrate, the reaction mixture gases, etc. [7]. The optimal process parameters require a lot of tests, which make possible to obtain a material with specific chemical and physical properties [7,8].

Raman spectroscopy is a basic tool for identifying different CVD diamond layers, thus, it is very helpful in finding the proper growth process 
Table 1. The position of Raman bands for different forms of carbon.

\begin{tabular}{cc}
\hline Raman shift $\left[\mathrm{cm}^{-1}\right]$ & Interpretation \\
\hline \hline 1580 & Monocrystalline graphite \\
1546 & Disordered graphite \\
$1500-1600$ & Amorphous carbon sp sp $^{2}$ G-band) \\
$1430-1470$ & Trans-polyacetylene laying in the grain boundaries \\
1345 & Amorphous carbon sp ${ }^{2}$ (D-band) \\
1332 & Diamond band \\
1220 & Disordered diamond \\
1150,1237 & Nanodiamond \\
$1100-1150$ & Trans-polyacetylene segments at the grain boundaries \\
\hline
\end{tabular}

parameters. This spectroscopy technique is a sensitive method to identify the variety of crystalline and disordered structures of a CVD structure, which may exist in different hybridization states of carbon, for example as $\mathrm{sp}^{2}$ or $\mathrm{sp}^{3}$ hybridization.

Different carbon structures, including diamond, in the Raman spectrum are visible in the spectral range of 200 to $3000 \mathrm{~cm}^{-1}$ (Table 1). The regions between 1520 and $1600 \mathrm{~cm}^{-1}$ as well as 1320 and $1360 \mathrm{~cm}^{-1}$ are defined as $\mathrm{G}$ and $\mathrm{D}$ bands, respectively [9].

\section{Experimental}

\subsection{CVD diamond growth process}

The diamond films with a thickness of about $1.5 \mu \mathrm{m}$, which are polycrystalline in nature, were deposited on silicon substrate by HF CVDtechnique. The filament operating at the temperature of $2300 \mathrm{~K}$ was placed $6 \mathrm{~mm}$ above the substrate. The mixture of methane vapor and hydrogen (from $1 \%$ to $2.5 \%$ of $\mathrm{CH}_{4}$ in $\mathrm{H}_{2}$ ) was used as a working gas. The parameters of the growth process were as follows: total pressure in reaction chamber: $6 \mathrm{kPa}$, substrate temperature: $1100 \mathrm{~K}$ and working gas flow rate: $100 \mathrm{sccm}$. In order to enhance the diamond nucleation density, the silicon substrate was scratched with diamond paste before the deposition process. After mechanical polishing with the diamond paste the substrate was washed in ultrasonic bath with methanol, chloroform and deionized water. The diamond film morphology has been studied by scanning electron microscope (SEM) JEOL JSM-820 operating at a voltage of $25 \mathrm{kV}$.

\subsection{Raman measurements}

All Raman spectra were recorded in air and at room temperature in backscattering geometry using Renishaw in Via Raman spectrometer. A tunable Ar ion laser was used as an excitation source of $488 \mathrm{~nm}$. The laser beam was tightly focused on the sample surface through a Leica 50x LWD microscope objective (LWD - long working distance) with a numerical aperture (NA) equal to 0.5 , leading to a laser beam diameter of about $2 \mu \mathrm{m}$. Spectral resolution was about $\pm 2 \mathrm{~cm}^{-1}$. The Raman scattering spectra of the diamond CVD layers were investigated in the spectral range of 1000 to $2000 \mathrm{~cm}^{-1}$. All data collection were analyzed using Renishaw WiRE 3.1 software by curve fitting method. The mix of Lorentzian and Gaussian functions was applied. Raman spectra were analyzed in detail for 13 samples obtained in deposition processes at different concentrations of $\mathrm{CH}_{4}$ working gas in the range of $1 \%$ to $2.5 \%$.

\section{Results and discussion}

With the increasing interest in technological applications of diamond, there has been an increase in the need to produce high-quality synthetic diamond crystals and films. Raman spectroscopy 
provides a fast, non-destructive method of characterizing these materials and evaluating their quality. In order to understand Raman spectra of CVD diamond films it was necessary to understand the Raman spectra of diamond, graphite and amorphous carbon films.

Single crystalline diamond has a very simple Raman spectrum, with a single sharp peak at $1332 \mathrm{~cm}^{-1}$ attributed to $F_{2 g}$ symmetry mode [9]. In the case of high ordered pyrolitic graphite we observed graphite peak at $1575 \mathrm{~cm}^{-1}$, which was attributed to the Raman active $\mathrm{E}_{2 \mathrm{~g}}$ symmetry mode [10].

Raman spectrum of amorphous carbon phase (Fig. 1) is composed of two peaks: the first one in the range of 1320 to $1360 \mathrm{~cm}^{-1}$, called D-band, and the second peak ranging from 1500 to $1600 \mathrm{~cm}^{-1}$, called G-band. The D line appears from $\mathrm{sp}^{2}$ hybridized carbon structure. The physical properties of amorphous carbon films strongly depend on the ratio of these two types of $\mathrm{C}-\mathrm{C}$ bonds. The ratio of $\mathrm{sp}^{3} / \mathrm{sp}^{2}$ carbon phases depends on deposition conditions and can be changed in a broad range from pure diamond to pure graphite.

The relative intensities of these vibrations vary depending on the crystallinity. The "G" peak corresponds to neighboring atoms moving in opposite directions perpendicular to the plane of the graphitic sheet. The "D" peak corresponds to atoms moving in radial directions in the plane of the graphitic sheet, similar to the breathing mode in benzene. The " $D$ ", disorder peak, appears as a result of dislocations in the lattice, deriving its name from the fact that they are "disorder-induced modes". As a result, the intensity of this peak relative to the "G" peak can be used to determine the degree of disorder in the sample. The structures of amorphous carbon phases depend on synthesis conditions and are reflected in their Raman spectra, i.e. the positions of $D$ and $G$ lines and their values of FWHM. Excellent review of Raman spectroscopy studies of amorphous carbon films has been done recently by Ferrari et. al. [11] and earlier by Schwan et. al. [12].

The identification of pure diamond or pure graphite by Raman spectroscopy is very simple

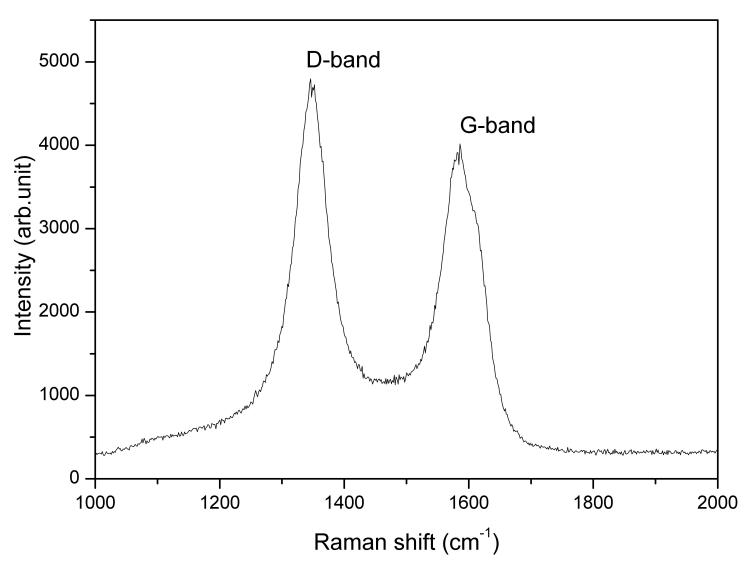

Fig. 1. Raman spectrum of amorphous carbon.

because both the structures are characterized by a single, sharp Raman line at about $1332 \mathrm{~cm}^{-1}$ and $1575 \mathrm{~cm}^{-1}$ for diamond and graphite, respectively.

In general, CVD diamond films consist numerous small diamond microcrystals surrounded by graphitic or amorphous carbon phase, situated mainly at grain boundaries [13]. With the increase in the mixture of methane vapor and hydrogen (from $1 \%$ to $2.5 \%$ of $\mathrm{CH}_{4}$ ) we observe decreasing grain sizes. Fig. 2 shows examples of SEM images of CVD diamond films morphologies for $1 \%$ (a) and $2.5 \%$ (b) $\mathrm{CH}_{4}$ concentration of methane used as a working gas, respectively.

In such case one can expect that for polycrystalline CVD diamond, the contributions to Raman spectrum from both $\mathrm{sp}^{3}$ and $\mathrm{sp}^{2}$ hybridizations of amorphous carbon should be observed in the Raman spectra except the diamond Raman line. The resulting Raman spectrum of CVD diamond film, in comparison to pure diamond, has more complicated character as it is shown in Fig. 3.

As it is clearly seen, the diamond Raman lines of all films are peaked almost at same positions but the $\mathrm{G}$ lines differ both in position and FWHM.

The Raman spectrum for CVD diamond films containing amorphous carbon phase includes overlapping peaks, and to resolve the bands it is necessary to apply some procedure. In the curve fitting procedure [1], an initial guess is generally made for some peak parameters in the interesting spectra region, and then the peak parameters are optimized 

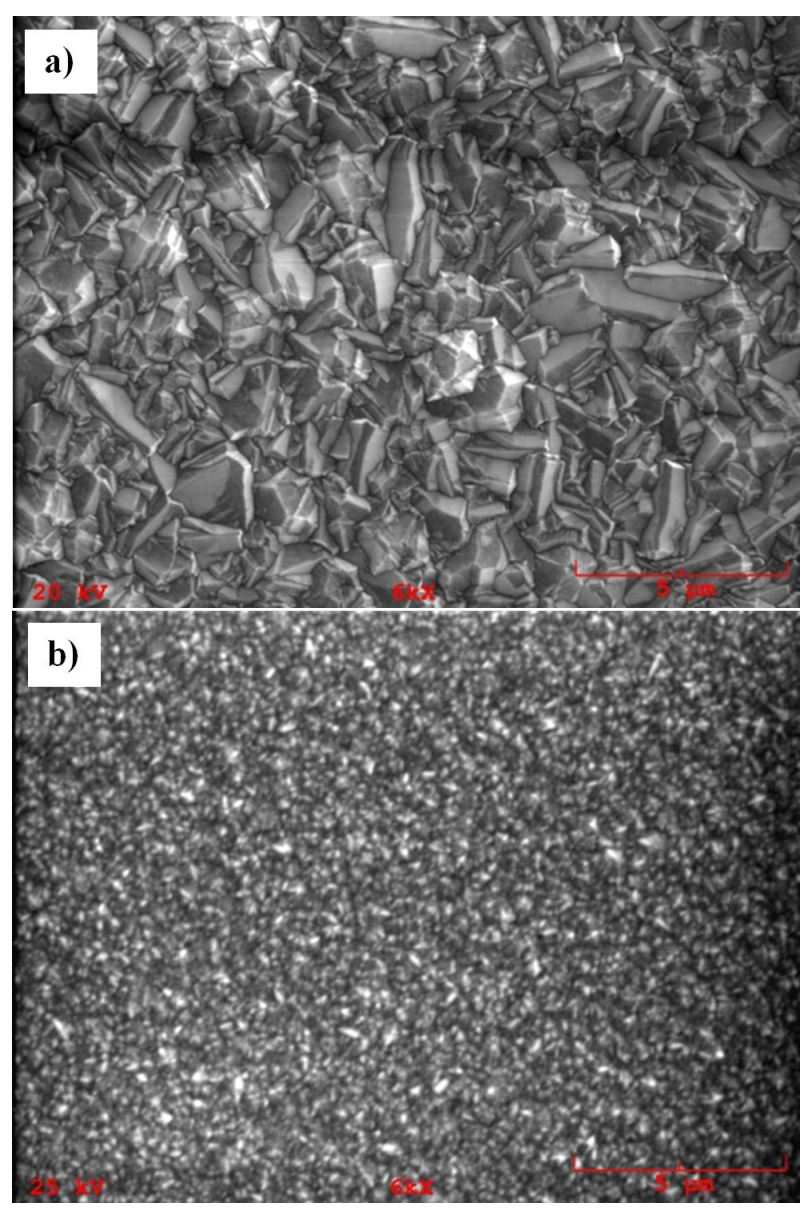

Fig. 2. Examples of SEM image of CVD diamond films morphologies.

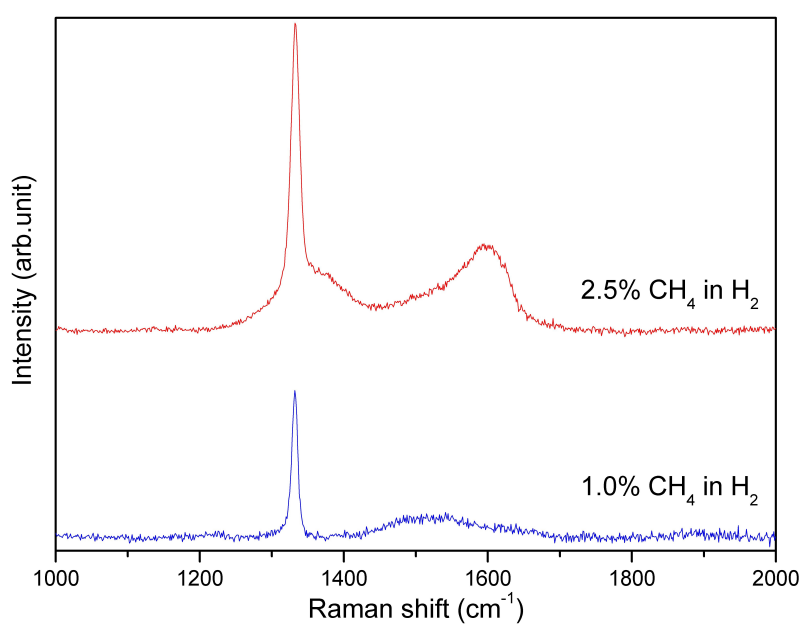

Fig. 3. Typical Raman spectra of two diamond films synthesized at different deposition conditions: with different $\mathrm{CH}_{4}$ working gas concentrations. to give the best fit to the measured data. The parameters refer to a mix of Lorentzian and Gaussian functions, which are believed to represent the shape of a single band.

Generally, five parameters are involved: the number of component peaks, their positions, shapes, half widths, and a baseline to allow for a background signal. In Fig. 4 it can be shown deconvolution of Raman spectrum of the CVD diamond films into diamond and amorphous carbon structure.

Such procedure allows us to characterize not only the diamond quality by FWHM of diamond Raman line but also the properties of amorphous carbon phase admixture by examination of $G$ and D lines parameters, such as their maxima position and FWHM.

As it has already been mentioned the ratio of the intensity of the diamond peak to the intensities of the "D" peak to " $G$ " peak $\left(\mathrm{I}_{\mathrm{D}} / \mathrm{I}_{\mathrm{G}}\right)$ can be used to estimate the amount of non-diamond phase, its crystallite size, thus, help to optimize parameters of diamond synthesis [14].

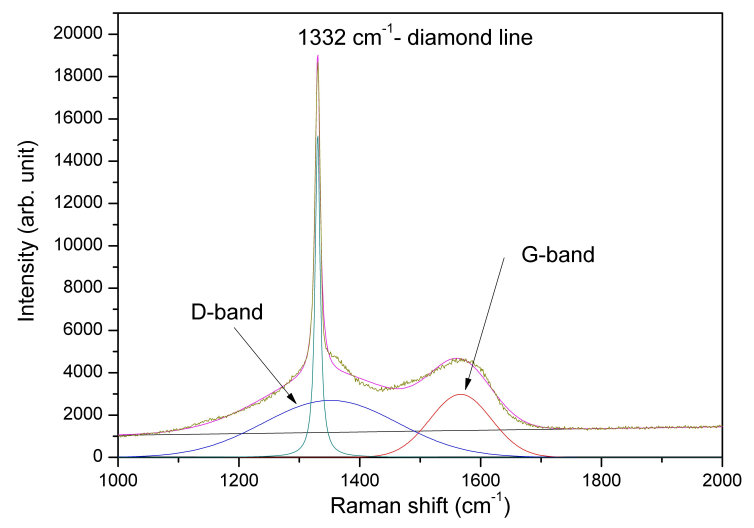

Fig. 4. An example of decomposition of CVD diamond Raman spectrum into pure diamond and amorphous carbon Raman components.

Lucchese et al. [18] proposed a three stage classification of disorders to simply assess the Raman spectra of carbons along an amorphization trajectory leading from graphite to tetrahedral amorphous carbon:

- stage 1: graphite to nanocrystalline graphite; 
- stage 2: nanocrystalline graphite to low $\mathrm{sp}^{3}$ amorphous carbon;

- stage 3: low $\mathrm{sp}^{3}$ amorphous carbon to high $\mathrm{sp}^{3}$ (tetrahedral) amorphous carbon.

In general, Raman spectra of amorphous carbon according to Ferrari et al. [11] depend on clustering of the $\mathrm{sp}^{2}$ phase, bond-length and bond-angle disorder, presence of $\mathrm{sp}^{2}$ rings or chains and on the $\mathrm{sp}^{3} / \mathrm{sp}^{2}$ ratio.

Ferrari et al. [15] suggested that the $\mathrm{G}$ band is attributed to some $\mathrm{sp}^{2}$ sites either in $\mathrm{C}=\mathrm{C}$ bonds or in aromatic rings, but the $\mathrm{D}$ band is due to the breathing mode of those $\mathrm{sp}^{2}$ sites in the rings, and to the long-range disordered graphite structure of the films. However, an increase in the D band intensity does not indicate increasing disorder in the film structure but may indicate the presence of various structural modifications in the films and may be not visible in the Raman spectrum of the CVD diamond film.

The $\mathrm{G}$ band (in general observed in almost all CVD diamond films) is due to the downward shift of the $1580 \mathrm{~cm}^{-1}$ graphite peak, and usually occurs between 1500 and $1600 \mathrm{~cm}^{-1}$, whereas the D-band generally, if it is visible in the spectrum, appears between 1320 and $1360 \mathrm{~cm}^{-1}[16,17]$.

In the present work we have analyzed $13 \mathrm{Ra}$ man spectra of CVD diamond films synthesized at different growth process conditions. Each Raman spectrum was deconvoluted into components as it is shown in Fig. 4.

The performed analysis allowed us to estimate the position and FWHM of each component of CVD diamond Raman spectrum as well as the ratio $\mathrm{I}_{\mathrm{D}} / \mathrm{I}_{\mathrm{G}}$ of $\mathrm{D}$ and $\mathrm{G}$ Raman bands. Depending on the $\mathrm{I}_{\mathrm{D}} / \mathrm{I}_{\mathrm{G}}$ ratios the $\mathrm{G}$-line has a maximum (in our case) in the range of 1510 to $1590 \mathrm{~cm}^{-1}$ and FWHM in the range of 90 to $190 \mathrm{~cm}^{-1}$ as it is shown in Fig. 5 .

FWHM of G-band always increases with disorder. Thus, combining $\mathrm{I}_{\mathrm{D}} / \mathrm{I}_{\mathrm{G}}$ and $\Gamma_{\mathrm{G}}$ allows one to discriminate between stages 1 (graphite to nanocrystalline graphite) or 2 (nanocrystalline graphite to low $\mathrm{sp}^{3}$ amorphous carbon) since samples in stage 1 and 2 could have the same $\mathrm{I}_{\mathrm{D}} / \mathrm{I}_{\mathrm{G}}$,
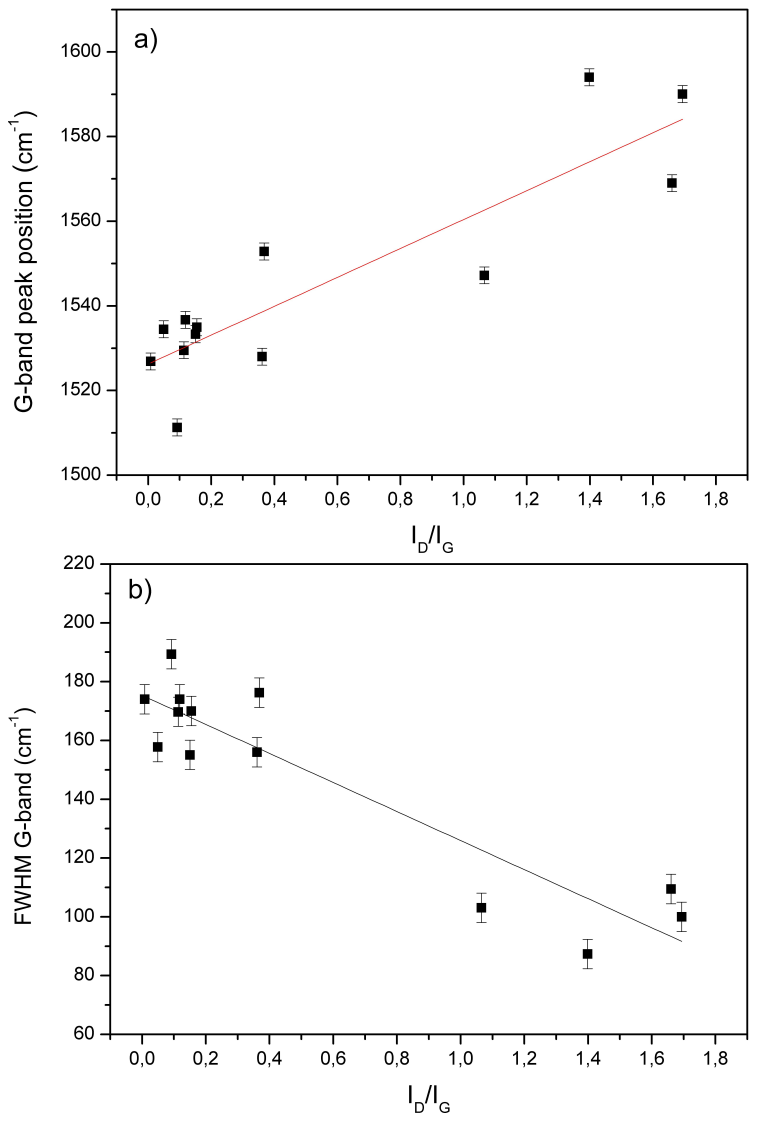

Fig. 5. The dependence of G-line position (a) and the corresponding FWHM (b) as a function of $\mathrm{I}_{\mathrm{D}} / \mathrm{I}_{\mathrm{G}}$ ratio.

but not the same FWHM which is much bigger in stage 2 [18].

Large values of FWHM of G-band, bigger than $90 \mathrm{~cm}^{-1}$, and G-line maximum shifted from $1510 \mathrm{~cm}^{-1}$ to $1590 \mathrm{~cm}^{-1}$ with decreasing $\mathrm{I}_{\mathrm{D}} / \mathrm{I}_{\mathrm{G}}$ ratios, indicate that amorphous carbon admixture in polycrystalline CVD diamond film has a structure described as stage 2 in the three stage amorphization model proposed by Ferrari et al. [11].

The values of FWHM of G-bands indicate that in-plane correlation length $\mathrm{L}_{\mathrm{a}}$ within the ordered graphite layers is below $1 \mathrm{~nm}$ and increases with decreasing of FWHM according to the general rule that FWHM increases with disorder of the carbon structure [18]. Fig. 6 presents FWHM of G-band as a function of G-band peak position. We can observe a linear dependence between FWHM and Raman band position of the G-band (decrease of FWHM 
Raman G-band, and increase in energy of this oscillation). In this case, we observe an increase in the defects in the carbon structure.
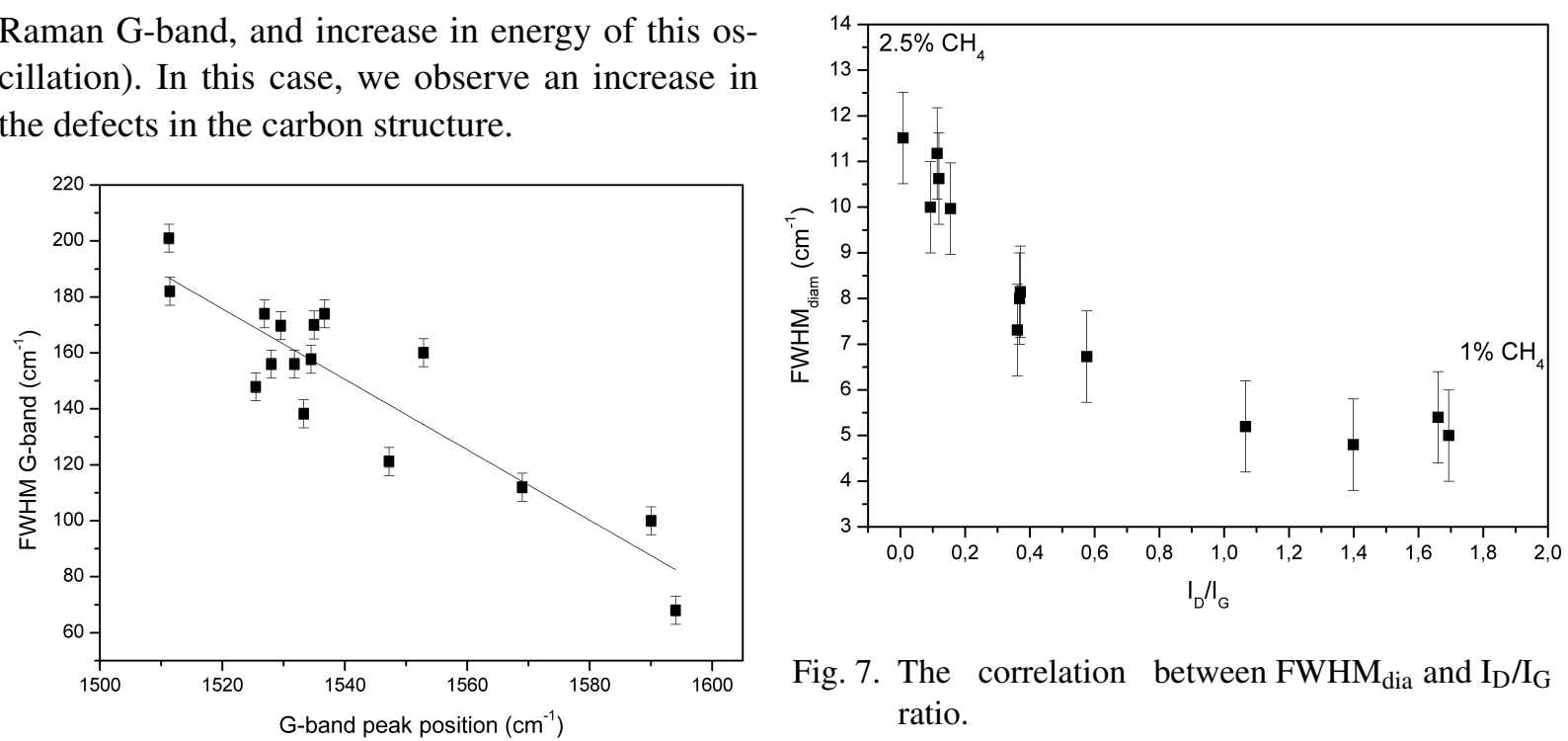

Fig. 7. The correlation between $\mathrm{FWHM}_{\text {dia }}$ and $\mathrm{I}_{\mathrm{D}} / \mathrm{I}_{\mathrm{G}}$ ratio.

Fig. 6. The dependence of FWHMG on G-band peak position.

For stage 2, according to amorphization trajectory [11], the distance $L_{D}$ between point defects increases as the ratio of $\mathrm{I}_{\mathrm{D}} / \mathrm{I}_{\mathrm{G}}$ increases and amorphous carbon structure is improving [19]. It has also been found the correlation (Fig. 7) between the amorphous carbon structure described by the $\mathrm{I}_{\mathrm{D}} / \mathrm{I}_{\mathrm{G}}$ ratio and diamond quality described by $\mathrm{FWHM}_{\text {dia }}$ of diamond.

The relation illustrated in Fig. 7 clearly shows that diamond quality strongly depends on the amorphous carbon structure, i.e. the higher value of $L_{D}$ $\left(\mathrm{L}_{\mathrm{D}} \approx \mathrm{I}_{\mathrm{D}} / \mathrm{I}_{\mathrm{G}}\right)$ the better diamond quality (lower value of FWHM $_{\text {diam }}$ ) [19].

\section{Conclusions}

The complexity of Raman spectra of diamond films with amorphous carbon admixture is discussed in this paper. The Raman spectroscopy is a useful nondestructive method to characterize different carbon structures, such as diamond, graphite and amorphous carbon. Structural analysis and knowledge of the carbon structure is an essential element related with the process for obtaining thin film structures of diamond by CVD method. Raman interpretation has to be performed with a great care due to the information being not visible

at the first look, lost as a result of the very broad Dline and G-line features. This makes the determination of cluster sizes and distributions based only on Raman spectroscopy very difficult. Even using both the $\mathrm{I}_{\mathrm{D}} / \mathrm{I}_{\mathrm{G}}$ ratio and the $\mathrm{G}$-line width, only a crude estimate of cluster sizes can be elucidated. Extensive analysis of Raman spectral parameters allowed us to find new relevant parameters to characterize the structure of the diamond structure and correlate them with the technological parameters.

\section{Acknowledgements}

This work was supported in part by the Research Project of Poznan University of Technology 06/62/DSPB/0415/2015.

\section{References}

[1] Fabisiak K., BanaszaK A., Kaczmarski M., KoZANECKi M., Opt. Mater, 28 (2006), 106.

[2] Worth C.J.H., Balmer R.S., Mater. Today, 11 (2008), 22.

[3] Kalish R., J. Phys D Appl. Phys., 40 (2007), 6467.

[4] Yang N., Uetsuka H., Osawa E., Nebel C.E., Nano Lett., 8 (2008), 3572.

[5] Greentree A.D., FAirchild B.A., Hossain F.M., PRAWER S., Mater. Today, 11 (2008), 22.

[6] FIELD J.E., The properties of diamond, Academic Press, Oxford, 1979.

[7] Anthony R.T., Clausing R.E., NATO Advanced Study Institute on Diamond and Diamond-like Films, Plenum, New York, 1991.

[8] Fabisiak K., Torz-Piotrowska R., Staryga E., Szybowicz M., PAProcki K., BanaszaK A., PoPielarski P., Mater. Sci. Eng. B-Adv., 177 (2012), 1352. 
[9] Prawer S., Nemanich R.J., Philos. T. R. Soc. A, 362 (2004), 2537.

[10] Tarrant R.N., WarschKow O., McKenzIE D.R., Vib. Spectrosc., 41 (2006), 232.

[11] Ferrari A.C., Robertson J., Philos. T. R. Soc. A, 362 (2004), 2477.

[12] SchWAN J., Ulrich S., Batori V., Ehrhardt H., Silva S.R.P., J. Appl. Phys., 80 (1996), 440.

[13] Teng K.Y., Chen H.C., Chiang H.Y., Horng C.C., Cheng H.F., Sankaran K.J., Tai N.H., LeE C.Y., LIN I.N., Diam. Relat. Mater., 24 (2012), 126.

[14] Fabisiak K., SzReiber M., UniszKiewiCZ C., Runka T., KASPROWiCz D., Cryst. Res. Technol., 45 (2010), 167.

[15] Ferrari A.C., Diam. Relat. Mater., 11 (2002), 1053.

[16] Knight D.S., White W.B., J. Mater. Res., 4 (1989), 385.
[17] APAKINA V.N., KarUZskiI A.L., Kogan M.S., Kvit A.V., Melnik N.N., Mityagin Y.A., Murzin V.N., Orlikovsky A.A., Perestoronin A.V., TChakenko S.D., VolchKov N.A., Diam. Relat. Mater, 6 (1997), 564.

[18] Lucchese M.M., Stavale F., Ferreira E.H.M., Vilani C., Moutinho M.V.O., CAPAZ R.B., ACHete C.A., Jorio A., Carbon, 48 (2010) 1592.

[19] Cancado L.G., Jorio A., Ferreira E.H., Dtavale F., Achete C.A., Capaz R.B., MoutinhP M.V.O., Lombardo A., Kulmala T.S., Ferrari A.C., Nano Lett., 11 (2011), 3190.

Received 2015-01-17

Accepted 2015-04-02 